\title{
The melanocyte in vertebrate evolution
}

\begin{abstract}
The evolution of the mammalian pigment cell, the melanocyte, is linked to the evolution of the neural crest cells, from which they derive. These cells allowed sessile filter-feeding ancestors to evolve into an active, mobile predator. As a part of this more mobile predatory system the melanocyte provided, at the periphery, both camouflage and protection from increased ultraviolet radiation exposure associated with this more active life-style.

It is proposed that these first cells originated from a progenitor of pigmented photoreceptors in an extinct organism. A pigment cell as an accompaniment of a photoreceptor cell in a two celled proto-eye. With changing environmental and photoreception needs the pigmented cell was given new roles. A more sophisticated camera-like eye was evolving from placodes in the head to provide more detailed photoreception. It, however retains genetic and mechanistic links to its ancient invertebrate relatives. The ability of the melanocyte to synthesise melanin has given it the opportunity to be co-opted to new applications in keeping with vertebrate evolution, moving from the central nervous system to its new sensory and protective role in the skin.
\end{abstract}

Keywords: melanocyte, neural crest, photoreception, proto-eye evolution, melanin
Volume 4 Issue 3 - 2020

\author{
David John Mackay Smith
}

University of Queensland,Australia

Correspondence: David John Mackay Smith, University of Queensland, I0 Mary Street Noosaville Queensland Australia, Email djmsmith@bigpond.com

Received: May 29, 2020 | Published: June 19, 2020

\section{Introduction}

The evolution of the mammalian pigment cell, the melanocyte, is linked to the evolution of the neural crest cell (NCC), from which they derive. These cells are a transient, multipotent, migratory cells of ectodermal origin. They are of relatively recent evolutionary origin, found only in vertebrate embryos and represent a highly multipotent population of embryonic stem cells. The particular features of tissues derived from neural crest cells allowed sessile filter-feeding ancestors to evolve into an active, mobile predator, rapidly ascending to the top of the food chain. In particular, the evolution of an animal with a head, containing an osseous jaw with dentition combined with a concentration of advanced sensory systems. This was supported by an improved endocrine system and effective neural transmission from peripheral sensors, allowing fast and reliable regulation of function by the central nervous system (CNS). As a part of this more mobile predatory system the melanocyte provided, at the periphery, both camouflage and a mechanism to monitor and counter the mutagenic effects of increased ultraviolet radiation exposure associated with this more active life-style.

\section{Neural crest origin}

Adameyko proposed that the neural crest originates from neuroepithelial progenitors of pigmented ocelli in Amphioxus-like animals. ${ }^{1}$ With changes in photoreceptive needs these progenitors of photoreceptors evolved into neural, glial and pigmentary progeny that gained the ability to migrate out of the central nervous system, providing a more useful role at the periphery.

Development of the neural crest begins in the dorsal neural tube where neuroepithelial progenitors undergo an epithelial to mesenchymal transition and delaminate into the sub-epithelial space Figure 1.

Inductive and specification events combine with navigational clues to guide the cells along various routes to their final, more peripheral destinations. The majority of neural crest-derived cells remain multipotent at the beginning of migration but gradually become more restricted in their differentiation potential on their journey to their destination. Recent studies suggest that some populations of NCC may become specified within the neural tube. ${ }^{2}$

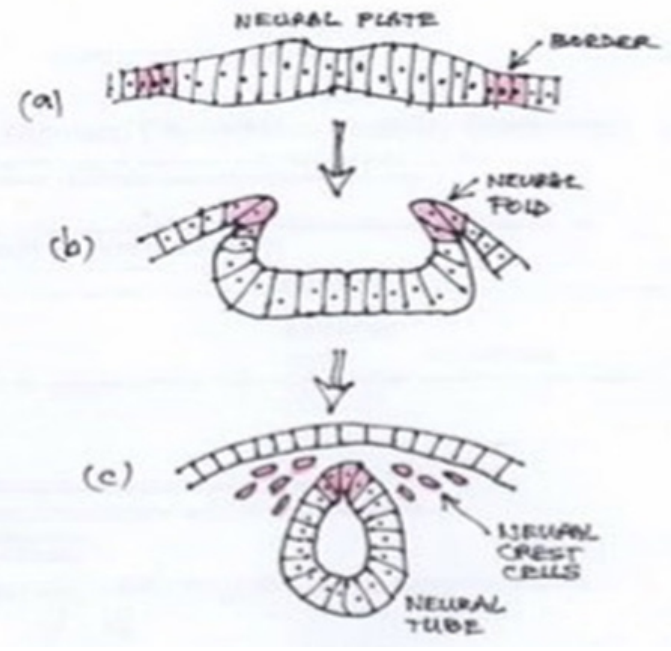

Figure I Neural crest formation. Diagram showing transverse section through the ectoderm layer. (a) Neural crest cells arising at the neural plate border. (b) As neurulation proceeds they are incorporated into dorsal neural folds. (c) Epithelial to mesenchymal transition in a rostro-caudal wave preceding migration to the periphery.

\section{Theories of neural crest evolution}

It is assumed that neural crest-like cells first appeared in a common ancestor of gnathostomes and cyclostomes because NCCs are found in both the lamprey and hagfish. However, there is a difference in the range of progeny indicating an incremental elaboration of a fully multipotent neural crest population through different vertebrate taxa. ${ }^{3}$ It remains unclear, however, if the first proto-neural crest cells were uni-or multipotent. At the level of the cytostomes, neural 
crest formation is governed by an evolutionarily conserved group of molecules, the neural crest gene regulatory network (NC-GRN), ${ }^{4}$ including neural crest specifiers and effectors and in control of delamination and migration.

In investigation of lower branches of the Chordata phylogenetic tree, tunicata and cephalochordate, cells were detected migrating from the neural tube into the body wall and siphon in Ectinascidia larvae (an ascidian tunicate), where they were differentiating into pigment cells $\mathrm{s}^{5}$ and these tunicates possessed some small part of the NC-GRN in trunk lateral cells. Unfortunately, the migratory neural crest-like cells were found to originate from trunk lateral cells rather than the CNS, and belonged to the mesodermal lineage. ${ }^{6}$ Other deuterostomes e.g. Sea urchins also acquire pigment cells of mesodermal origin. Therefore, these cells represent parallel evolution rather than being neural crest homologous tissue.

Other investigators, however, proposed that melanin-containing cells in ascidian CNS might be evolutionary precursors of neural crest-derived melanocytes. ${ }^{8}$ Abitua et al demonstrated that an ascidian, Ciona, possesses a cephalic melanocyte lineage that can be reprogrammed into a migratory multipotent population. This lineage expresses neural crest specification genes and gives rise to melanocytes of light-detecting ocellus and otolith. ${ }^{9}$ They also showed that signaling pathways governing specification of the ascidian ocellus are conserved with specification events in vertebrate neural crest lineage, including Forkhead transcription factor (FoxD) -mediated repression of melanocyte inducing transcription factor (MITF). It is possible that the FoxD/MITF relationship represents an ancient interaction, in photosensitive structures, that evolved before the split of chordates from other animals, without a connection to neural crest. They proposed that the presence of neural crest-specific regulatory proteins in ascidian pigmented ocellus lineage, as in the cephalic melanocytes of Ciona, represents a rudimentary neural crest. ${ }^{10}$ This study supports the hypothesis that mesenchymal fates were a late acquisition during neural crest evolution. ${ }^{11}$ However, it is still not clear how multiple mesenchymal fates are enabled upon migration of targeted cells outside the CNS. ${ }^{1}$

Amphioxus (lancelet) the basic contemporary chordate, does not have a neural crest, and neurulation occurs completely differently to higher vertebrates and cyclostomes. Non-neural ectodermal sheets migrate above the invaginating neural plate towards the dorsal midline where they fuse, however, these non-neural epithelial cells never migrate as individuals. ${ }^{12}$ Linda and Nicholas Holland suggested that these sheets may represent a homologous tissue to vertebrate neural crest. ${ }^{13}$ Delaminating, migrating NCCs were present in ancestors of hagfish and other vertebrates in the Cambrian, 500 million years ago. ${ }^{14}$ Neural crest specifiers are not expressed in the neural plate of Amphioxus, although, they produce a melanin-based pigment, so some neural crest-based programs are conserved. Based on this, Nikitina et al suggested that several independent cell types with diverse differentiation potential may have collectively assembled a presumptive tissue at the neural plate border giving rise to the future multipotent NCC. ${ }^{15}$ This is opposed to previous ideas that a unipotent migrating proto-NCC gives rise to multipotency later. An alternative hypothesis suggested that glial cells from Amphioxus dorsal roots were the origin and might be considered similar to the neural crestderived Schwann Cell Precursor. ${ }^{16}$

Adameyko supports the concept that the proto-neural crest evolutionarily originated from neuroepithelial cells and the neuroepithelial fates to be the most ancient and primordial in the
NCC lineage. He further proposes that the first NCCs originated from the multipotent progenitor of pigmented photoreceptors in an extinct lancelet-like organism.

\section{The proto-eye}

If it can be assumed that the melanocyte is partially, at least, a cell of photoreception, rather than looking at the evolution of the neural crest, there may be an alternate view from examining the evolution of the eye. While morphological comparison of animal photoreceptor cell types led to the view that there were multiple paths of alternative evolution, the molecular conservation of the paired box 6 gene ( $\operatorname{pax} 6$ ) eye-specifying cascade has indicated that they evolved from a simple precursor, the proto-eye. Arendt has the view that retinal ganglion, amacrine and horizontal cells are evolutionary sister cell types that evolved from a common rhabdomeric photoreceptor cell precursor. ${ }^{17}$ Gehring and Ikeo have suggested a two-cell proto-eye, made of one photoreceptor cell and one pigment cell, ${ }^{18}$ resembling the twocelled eye represented in primary ciliary larvae such as polychaete trochophore. ${ }^{19}$ Figure 2.
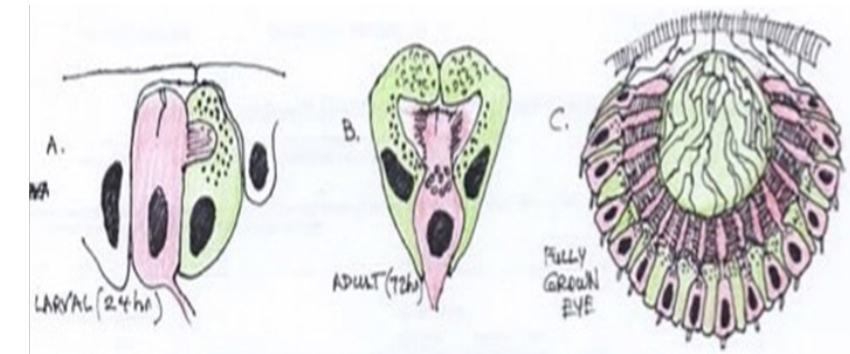

Figure 2 Two-celled larval eye and prototype pigment-cup eye with rhabdomeric photoreceptors in Playnereis dumerilli. Note the nuclei of the photoreceptor cells positioned behind the shielding pigment granules.

Red-Rhabdomeric photoreceptor cell; Green-pigment cells. (Adapted from Arendt $\left.1999^{17}\right)$.

This points to a very early evolutionary relationship between photoreception and pigmentation. A relationship that has persisted into modern mammalian anatomy. The proto-eye is represented in many invertebrate groups as the simple pigment cup eye-photoreceptors embedded in a cup-shaped layer of shaded pigment. It appears that the animal eye relies on the basic principle of photoreceptor in the vicinity of dark shielding pigment. The receptor converts light (Photon stream) into intracellular signaling. The dark pigment (melanin in vertebrates) reduces photon scatter and orients the direction optimally sensitive to light. Not only can melanin absorb photons, but also electrons and free radicals such as reactive oxygen species, and so acting as a chemical filter for sensitive cells such as the light sensing organs found in tunicates and amphioxus.

All photoreceptor cells use a vitamin A-based light sensitive photopigment, comprising a chromophore and an apoprotein, opsin, a 7 transmembrane receptor. Phylogenetic analysis suggests that all opsins trace back to one opsin precursor molecule that predates bilaterians. ${ }^{17}$ Absorption of a quantum of light by the rhodopsin molecule converts all trans retinal that is covalently bound to the opsin protein into 11-cis retinal. This causes a conformational change in the protein. Phototransduction requires the binding of photoactivated opsin to the $\alpha$ subunit of a G-protein, that amplifies the signal and results in an electrical nerve impulse. Quenching of the phototransmission cascade involves rhodopsin kinase that phosphorylates the opsin and Arestin that competes with the $\alpha$ subunit for binding to the opsin..$^{20}$ 


\section{Pax 6 gene and eye morphogenesis}

Darwin in his 'The Origin of Species' admits the difficulty of suggesting that natural selection could produce "an organ as perfect as the eye." He postulated an imperfect proto-eye "from which a more wonderful visual organ may have arisen by mutation and natural selection." Early proponents of Darwinism postulated that photoreceptor organs originated independently in over 50 phyletic lines. However, the observation that all metazoans share the same visual pigment, rhodopsin, supports a monophyletic origin from a common ancestral precursor. There is also ancient pax6 requirement for the specification of a pre-bilaterian photoreceptor cell precursor. ${ }^{18}$ A pax6 ortholog is expressed in ocelli and in vertebrates, and pax6 expression is still required for virtually all photoreceptor retinal cell types. ${ }^{21}$ The Pax6 conservation through photoreceptor cell and eye development led to the idea that Pax 6 might be the universal master control gene for eye morphogenesis and evolution. ${ }^{22}$ Pax 6 genes are expressed in developing eyes of planarians which is close to the Darwinian prototype.

In cnidarians the situation is less clear. Genes found are either precursors of Pax6 or have diverged too far to be identified as homologs. However, Pax 6 was present in the last common ancestor of all these triploblastic phyla, much like the rhodopsin gene. ${ }^{18}$

\section{Evolution of different types of eyes}

Rhodopsins are present in some bacteria, and some of these proteins serve a sensory function. However, there is little sequence conservation between these and rhodopsins of higher organisms, even though both are structurally similar membrane proteins. The unicellular alga, Chlamydomonas, has developed a visual system, the eyespot, that allows it to detect light intensity and direction. A carotenoid-containing vesicle operating as an interface and dependent on a reflector. It has a maximum action spectrum around $550 \mathrm{~nm}$, similar to Rhodopsin, and it shows some sequence homology to invertebrate rhodopsin. However, it does not have a similar receptor, looking more like an ion channel, having probably diverged from animal opsin early in evolution.

Originating from a pre-Cambrian prototype, the various types of eye are thought to have evolved by recruiting additional genes into eye development pathways. Pax 6 illustrates that novel genes can be generated in the course of evolution by recombining parts of preexisting genes in a process Jacob called evolutionary tinkering. ${ }^{23}$ In cnidarians the eyes directly connect to muscles in the tentacles, suggesting that the eye evolved as an information-gathering organ before the brain, the information-processing organ.

\section{Rhabdomeric and ciliary photoreceptors}

Photoreceptors in metazoans can be grouped into 2 classes. Both use some form of visual pigment protein, opsin, together with 11cis retinaldehydes to absorb light and activates a $G$ protein cascade, resulting in opening or closing of ion channels. All photoreceptor cells enlarge the membranous surface for the storage of photopigment. Rhabdomeric photoreceptor cells do so by folding the apical cell surface, creating microvilli, while ciliary cells fold the ciliary membrane. ${ }^{24}$ Both receptor cell types exist in many bilaterian groups Figure 3 .

Genetic studies show that both receptor types emerged from a single precursor and were present very early in the evolution of metazoans. The opsins used by photoreceptors also divide into clear classes.
Microvilli express r-opsins and ciliary use c- or G -opsin subfamily. This separation of photoreceptor types and family of opsins is very old, likely present before the emergence of the bilateria. ${ }^{25,26}$ After splitting apart, both the new sister cell types specialised on different intercellular messenger pathways, phospholipase C (PLC) versus phosphodiesterase (PDE), respectively Figure 4. Another example of both receptor types sharing an early common evolutionary origin is seen in the vertebrate eye. The comparison of molecules involved in the specification and differentiation of rhabdomeric photoreceptor cells and with those of the different cell types in the vertebrate retina has revealed many resemblances, not with the rod and cone ciliary receptor cells, but with the retinal ganglion cells (RGCs). This has led to the proposal that RGCs and rhabdomeric cells are homologous cell types that trace back to the same precursor cell type in urbilateria. ${ }^{19}$

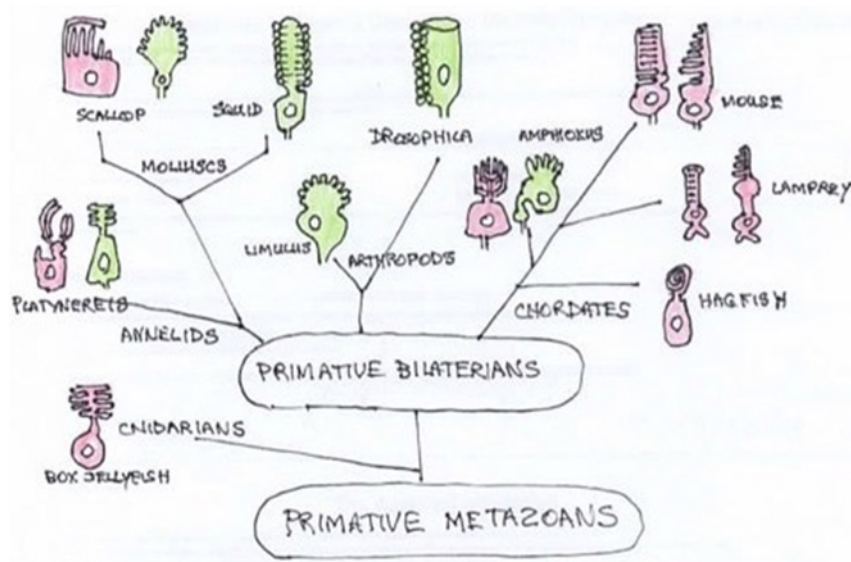

Figure 3 Phylogenetic tree of metazoans. Photoreceptor types in principle eyes are illustrated as ciliary (red) or microvilli (green).Vertebrates have ciliary receptors but have retained the rhabdomeric transduction cascade in ganglion cell in the eye. (Adapted from Fain et al.,) ${ }^{29}$

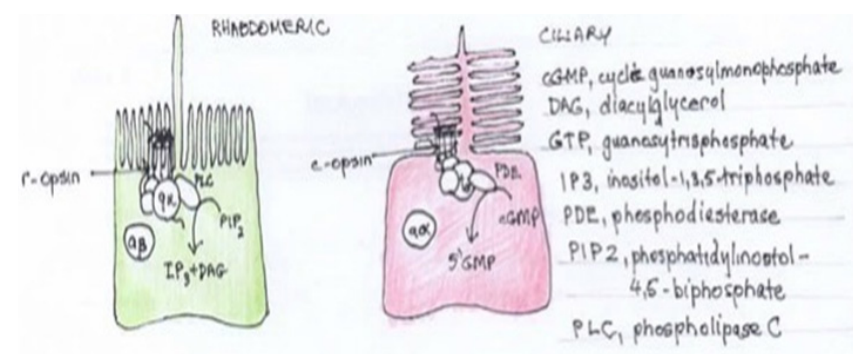

Figure 4 Rhabdomeric versus ciliary photoreceptors. R-opsin receptor and phospholipase $C$ transmitter versus c-opsin and phosphodiesterase transmitter, respectively.

In the most basal chordate, melanopsins are found in rhabdomeric photoreceptors represented by the Hesse organs of Amphioxus, which consists of a photosensory cell and a melanin-containing cup cell. The presence of a melanin together with melanopsin-mediated light sensitivity in a pigment cup cell of the lancelet strongly resembles the features of the melanocyte. Expression of melanopsin with a PLC-based phototransmission pathway suggests that melanocytes are related to invertebrate rhabdomeric photoreceptors. Other contemporary examples are sponge larva photosensory cells, cnidarian photoreceptors and ascidian ocellus. To account for the combination of features present in the melanocyte, it implies that the ancestral protochordate photosensitive structure already contained several distinct cell types, including ciliary and rhabdomeric 
photoreceptors originating from the same progenitor cell type located in the neural ectoderm. Abitua et al demonstrated that the fate of the tunicate ocellus is controlled by wingless-related integration site (Wnt)signaling, and Fox D repressed MITF in a similar fashion to fate specification of the melanocyte lineage, strengthening the evolutionary connection between pigmented photosensory lineage in protochordates with melanocytes. ${ }^{10,27}$ MITF is also expressed in the ciliary eyes of cubozoan jellyfish Tripedalia cystophora (box jellyfish), a very ancient and primitive diploblastic animal, but the cnidarian is thought to represent parallel development rather than having a common ancestor. ${ }^{28}$

At some point prior to the cnidarian-bilaterian split the r-opsin family and microvilli receptors emerged with their transduction mechanism based on phospholipid $\mathrm{C} / \mathrm{Ca} 2+$ signaling. Both ciliary and microvilli classes are still found in most phyla, often with one kind subserving spatial vision in the image forming eyes and the other adopting an accessory role. In most protostomes, including arthropods, mollusks and annelid, microvilli dominant. Chordates, including vertebrates, have ciliary receptors with c-opsin and GMPbased cascades. R-opsin /PLC transduction is retained in melanopsincontaining retinal ganglion cells providing the papillary response.

Microvilli are retained because they support vision in dim light with large responses to a single photon. This allowed emerging bilaterian species occupy a range of environments from deep sea to surface and eventually terrestrial habitats. The microvilli organisation not only promotes photodetector in dim light but also helps reduce their gain by orders of magnitude to allow function in bright light. By comparison, ciliary receptors of invertebrates detect light over a restricted range of intensity with reduced sensitivity and inability to function in both dim and bright light. They came to dominate in chordates with the invention of the high sensitivity rods, for scotopic vision in dim light, supplementing the low sensitivity cone-like receptors, for fast accurate photopic vision in bright light. Chordates deploying both rods and cones to cover the full range of light intensity. ${ }^{28}$

\section{From protochordate to vertebrate scenario}

Despite the adoption of microvilli receptors by many invertebrates, ciliary receptors were retained by most taxa, functioning as diurnal cone-like detectors in bright light but also possibly involved in circadian rhythm control. ${ }^{29}$

Lancelets represent a side branch of the chordate phylogenetic tree and are not direct ancestors of vertebrates but Amphioxus can be a model reflecting the early primitive condition of chordate organisation. The lancelet has 4 distinct photoreceptive modules embedded in the CNS.

- Frontal ciliary eye- homologous to the vertebrate eye, for orientation.

- Dorsal lamellar body- also ciliary. A proto-pineal gland controlling circadian rhythm.

- Dorsal rhabdomeric Joseph cells. Ocelli monitoring position in the burrow.

- Numerous ventral Hesse organs, also rhabdomeric ocelli.

Light at the ocean floor could penetrate the thin translucent body of the lancelet to the internal photoreceptors within the CNS, but with increasing size, photosensitive cells were required at the periphery. Migration of these tissues may have been accompanied by sensory neurons and glia, coming from the same lineage. Progressive cephalisation with accompanying development of anterior sensory appendages, particularly the eye, would have allowed movement out of the burrow, and the pursuit of a free swimming, more predatory behavior, making the subcutaneous ocellus redundant. However, photosensitivity and ability to produce melanin were likely maintained in the lineage, allowing light controlled camouflage and protection from UV radiation. An extinct cephalochordate, Pikaia gracilens, has been uncovered. It is larger than lancelets, has a head with appendages and a high flat body with fins suggesting locomotion and predication. Changes in lifestyle leading to changes in photoreception figure 5.

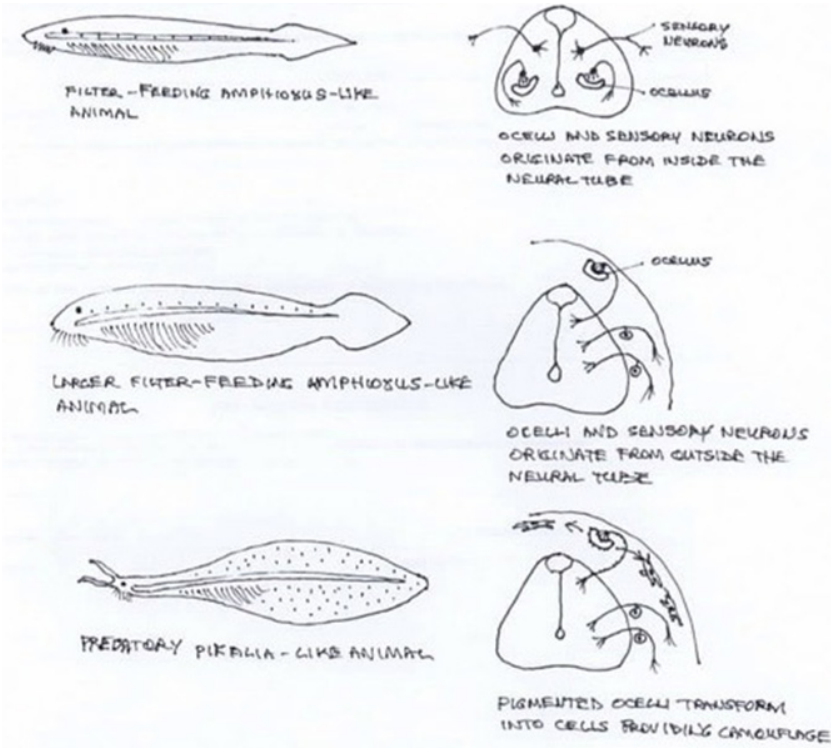

Figure 5 Evolutionary scenario of photoreceptors within the CNS giving rise to neural crest elements in a lineage of early chordate animals. (Adapted from Adameyko').

\section{Melanocyte photo reception}

It has been recognised that ultraviolet radiation (UVR) causes DNA damage that leads to transcriptional activation of melaninproducing enzymes, epidermal melanocytes acting to protect the vertebrate from carcinogenic effects of the UVB radiation by a delayed skin pigmentation, taking days to manifest. In contrast, UVA causes oxidative effects leading to immediate pigment darkening (IPD) within minutes. Nadine Wicks et al identified a receptor protein expressed in epidermal melanocytes as the visual pigment rhodopsin and phototransmission involving phospholipid C (PLC) causing calcium mobilisation and early melanin synthesis. Cellular melanin continued to increase in a retinal- and calcium-dependent manner up to 5 -fold after 24 hours. ${ }^{30}$ This effect maybe analogous to the dermal melanocytes of fish andamphibia that are also light sensitive, responding to changes in light intensity with changes in the extent of pigment aggregation that aid in camouflage and social communication.

\section{Which came first}

Which came first, rhodopsin expression in pigment cells or in eye photoreceptor cells? The light response of pigment cells serves for colour adaption and what are colour changes good for if not for eyes to see them, so this would suggest the eye as a photoreceptor. Ancestral 
vertebrates, equipped with eyes expressing vertebrate opsins, may have retained invertebrate opsins for light sensitivity in cells outside the eye. Pigment cells may then have recruited an invertebratetype opsin gene for its own light-sensing needs. Alternatively, light-dependent intracellular pigment redistribution may have been invented not for colour adaptation but for thermoregulation and photoprotection. Because pigment cells and photoreceptor cells may not just share a rhodopsin but also pigment granules, pigment cells may have been an evolutionary precursor to photoreceptors. Invertebrate PRCs often contain pigment granules of their own, and some e.g. Grass shrimp, Palaemontes pugio, controls light scattering by redistribution of pigment granules in response to light, in the same way as melanophores. In vertebrate eyes, screening function is provided by the neighbouring pigment cell (containing the pigment granules) which develop from the same tissues as PRCs. The skin pigment cells (melanopsin cloned) are neural crest not neural tube from which PRCs develop. Both are ectoderm, so both could have common evolutionary origin. The two cell types, being evolutionarily related, could share expression of a wider range of proteins than rhodopsins, including regulatory, structural and signalling molecules. Rhodopsin expressing pigment cells in the skin of an ancestral animal could have been well poised to provide a molecular package from which the eye finally arose.

\section{Conclusion}

The first evidence of a pigment cell associated with photoreception is as a humble accompaniment of a photoreceptor cell in a two celled proto-eye of urbilateria. Its presumptive function was to reduce light scatter to aid localisation by the photoreceptor cell. As the early marine chordates became larger, photoreceptor function was required to move from the CNS to the periphery. This change was accompanied by cephalisation and the development of a more sophisticated sensory apparatus emanating from the head. This general period of change from sessile filter-feeder to active predator was enabled by elaboration of the neural crest, providing mobility and multipotency to elements of the nervous system with the development of the peripheral nervous system allowing communication between peripheral sensors and the CNS within the vertebrate taxa. The pigmented cell was given a new role in keeping with changing environmental and photoreception needs. A more sophisticated camera-like eye was evolving from placodes in the head to provide more detailed photoreception.

The pigment cell was now providing skin pigmentation for camouflage, social signalling and sexual attraction. It was also able to provide protection for the keratinocytes in the skin with increased UVR exposure related to their active life-style. It also retained the rhabdomeric photoreceptor and photo transmission components to provide signalling in relation light and UV radiation. A genetic and mechanistic link to its ancient invertebrate relatives.

Melanin is ubiquitous in living organisms, now and in antiquity, and is represented in all kingdoms, plant, animal and fungi. This demonstrates the wide range of potential applications of this versatile chemical. The ability of the melanocyte to synthesise melanin has given it the opportunity to be co-opted to new applications with vertebrate evolution.Movement from the CNS to its new sensory and protective role, in a synergistic relationship with the keratinocyte, in the skin. Its ancestral progenitors also involved in evolution of the eye.

\section{Conflicts of interest}

The author declares that there is no conflicts of interest.

\section{Acknowledgments}

None.

\section{Funding}

None.

\section{References}

1. Ivashkin E, Adameyko I. Progenitors of the protochordate ocellus as an evolutionary origin of the neural crest. Evo Devo. 2013;4(12):1-15.

2. Krispin S, Nitzan E, Kalchein C. The dorsal neural tube: a dynamic setting for cell fate decisions. Dev Neurobiol. 2010;70(12):796-812.

3. Hall B, Gillis J. Incremental evolution of the neural crest, neural crest cells and neural crest-derived skeletal tissue. J Anat. 2013;222(1):19-31.

4. Suka-Spengler T, Meuliman D, Jones M, et al. Ancient evolutionary origins of the neural crest gene regulatory network. Dev Cell. 2007;13(3):405-420.

5. Jeffery W, Stuckler A, Yamamoto Y. Migratory neural crest-like cells form body pigmentation in a chordate embryo. Nature. 2004; 431(7009):696699.

6. Jeffery W, Chiba T, Krajka F, et al. Trunk lateral cells are neural crestlike cells in the ascidian Ciona intestinalis: insights into the ancestry and evolution of the neural crest. Dev Biol. 2008;324(1):152-160.

7. Ruffins s, Ettensohn C. A clonal analysis of secondary mesenchyme cell fates in the Sea Urchin embryo. Dev Biol. 1993;160(1): 285-288.

8. Baker C, Bronner-Fraser M. The origins of the neural crest. Part II: an evolutionary perspective. Mech Dev. 1997;69(1-2):13-29.

9. Nishida H, Satoh N. Determination and regulation in the pigment cell lineage of the ascidian embryo. Dev Biol. 1989;132(2):355-367.

10. Abitua P, Wagner E, Navarrete I. et al. Identification of a rudimentary neural crest in a non-vertebrate chordate. Nature. 2012;492(7427):104107.

11. Shimeld S, Hollan P. Vertebrate innervations. Pro Natl Acad Sci USA. 2000;97(9):4449-4452.

12. Holland N, Panganiban G, Henyey E, et al. Sequence and developmental expression of AmphiDII, an amphioxus distal-less gene transcribed in the ectoderm, epidermis and nervous system: insights into evolution of craniate forebrain and neural crest. Development. 1996;122(9):29112910.

13. Holland L, Holland N. Evolution of the neural crest and placodes: amphioxus as a model for the ancestral vertebrate. J Anat. 2001; 199(12): $85-98$.

14. Shu D, Morris S, Hau J, et al. Head and backbone of the early Cambrian vertebrate Haikouichthys. Nature. 2003;421:526-529.

15. Nikinita, V., Bronner-Fraser, M. Gene regulatory networks that control the specification of neural crest cells in the lamprey. Gene regulatory mechanisms. 2009; 1789(4):274-278.

16. Baker $\mathrm{C}$. The evolution and elaboration of vertebrate neural crest cells. Curr Opin Genet Dev. 2008;18(6):536-543.

17. Arendt D. Evolution of eyes and photoreceptor cell types. Int J Dev Biol. $2003 ; 47: 563-571$.

18. Gehring, W, Ikeo K. Pax 6: mastering eye morphogenesis and eye evolution. Trends Genet. 1999;15:371-377.

19. Arendt D, Tessmar K, De Campos-Baptista M, et al. Development of pigment-cup eyes in the polychaete Platynereusdumerilli and conservation of larval eyes in bilateria. Development. 2002;129(5):1143-1154. 
20. Krupnick J, Gurevich, V, Benovic J. Mechanism od quenching of phototransmission for phosphorhodopsin. J BiolChem. 1997; 272(29):18125-18131.

21. Marquardt J, Ashey-Padan R, Rejewski N, et al. Pax 6 is required for the multipotent state of retinal progenitor cells. Cell. 2001;10(1):43-55.

22. Quiring R. et al. Homology of the eyeless gene of Drosophila to the small eye gene in mice and Aniridia in humans. Science. 1994;265:785-789.

23. Jacob F. Evolution and tinkering. Science. 1997;196:1161-1166.

24. Eakin R. Continuity and diversity in photoreceptors. In: Visual Cells in Evolution. J Westfall, editor. Raven Press. New York: 1982;91-105.

25. Suga H, Schmid V, Gehring N. Evolution and functional diversity of jellyfish opsins. Curr Biol. 2008;18(1):51-55.
26. Arendt D, Willbrodt J. Reconstructing the eye of urbilaterian. Phil Trans Roy SocLond. 2001;356(1414):1545-1563.

27. Hari L, Miescher I, Shakhova V, et al. Temporal control of neural crest lineage by Wnt/ $\beta$-catenin signalling. Development. 2012;139(12): $2107-$ 2117.

28. Koznik Z, Ruzickova J, Jonasova K, et al. Assemby of the cnidarian camera-type eye from vertebrate-like components. Proc Natl Acad Sci USA. 2008;105(26):8989-8993.

29. Fain, G, Hardie R, Laughlian S. Phototransduction and the evolution of photoreception. Curr Biol. 2010;20:114-124.

30. Wicks N, Chan J, Laughlin, J, et al. UVA phototransduction drives early melanin synthesis in human melanocytes. Curr Biol. 2011; 21(22):19061911. 